\title{
L'Alliance française : les années Foncin (1883-1914). Contexte, naissance, mutations
}

\section{Ivan Barko}

\section{(2) OpenEdition \\ Journals}

Édition électronique

URL : https://journals.openedition.org/dhfles/2948

DOI : $10.4000 /$ dhfles.2948

ISSN : 2221-4038

Éditeur

Société Internationale pour l'Histoire du Français Langue Étrangère ou Seconde

Édition imprimée

Date de publication : 1 décembre 2000

Pagination : $90-115$

ISSN : 0992-7654

Référence électronique

Ivan Barko, «L'Alliance française : les années Foncin (1883-1914). Contexte, naissance, mutations », Documents pour l'histoire du français langue étrangère ou seconde [En ligne], 25 | 2000, mis en ligne le 10 septembre 2017, consulté le 27 mai 2021. URL : http://journals.openedition.org/dhfles/2948 ; DOI : https://doi.org/10.4000/dhfles.2948

Ce document a été généré automatiquement le 27 mai 2021

(C) SIHFLES 


\title{
L'Alliance française : les années Foncin (1883-1914). Contexte, naissance, mutations
}

\author{
Ivan Barko
}

\section{Introduction ${ }^{1}$}

C'est en 1983, à la veille du centenaire de l'Alliance française de Paris, que le premier (et jusqu'ici le seul) ouvrage consacré à l'histoire de cette institution a été publié. Basé sur le dépouillement du Bulletin de l'Alliance française ${ }^{2}$, ce volume, signé Maurice Bruézière ${ }^{3}$, passe en revue les principales étapes de la formation et de l'évolution de l'Alliance dans le monde et à ce titre il constitue le point de départ de recherches plus fouillées ainsi que de tout commentaire analytique sur les origines et le développement de l'Alliance.

Les archives de l'Alliance française de Paris, emportées par les Allemands pendant la guerre et considérées pendant longtemps comme perdues à jamais, ont été récemment retrouvées à Moscou ${ }^{4}$. Nul doute qu'un jour, que nous espérons prochain, rapatriées en France, elles seront mises à la disposition des chercheurs. Alors la publication d'une histoire définitive de l'Alliance française deviendra possible.

En attendant ce jour, nous devons nous contenter de travaux d'une portée plus limitée et proposant des conclusions provisoires : c'est tout ce que nous espérons accomplir ici. Nous esquisserons les grandes lignes de l'évolution de l'Alliance de sa création en 1883-84 jusqu'à la Première Guerre mondiale. Sur la base du livre de Maurice Bruézière et nous inspirant de travaux divers sur la mission civilisatrice de la France, sa politique linguistique et culturelle et le débat colonial pendant l'époque $1900^{6}$, nous tenterons de situer la genèse de l'Alliance dans son contexte historique et de décrire les mutations qu'elle a opérées, tant au niveau de son idéologie qu'à celui de son action pratique, au cours des trente années pendant lesquelles Pierre Foncin présida à ses destinées. 
Plus que des faits nouveaux, nous pensons pouvoir faire ressortir, par des éclairages nouveaux, des aspects jusqu'ici méconnus de l'histoire de la première Alliance française.

\section{Contexte}

\section{Expansion coloniale et politique linguistique}

A l'aube des années quatre-vingt du XIXe siècle la droite française a perdu les élections législatives. Pendant les cinq années suivantes, Jules Ferry (de septembre 1880 à novembre 1881), puis Gambetta, Freycinet, Duclerc et Fallières et ensuite encore une fois Ferry (1883-85) se trouvèrent successivement à la tête du gouvernement. Dans le domaine colonial et extérieur, ainsi que dans celui de l'éducation, ces présidents du Conseil de gauche poursuivaient une politique sensiblement identique. De plus, chacune de ces personnalités, lorsqu'elle n'était pas à la tête du gouvernement, était chargée d'un ministère dans le cabinet du président du Conseil du moment, de sorte que, malgré l'instabilité apparente du système, ces années (allant de 1880 à 1885) peuvent être considérées comme une période cohérente d'action politique.

L'Alliance française a été fondée pendant cette période. Le maître d'œuvre fut Pierre Foncin, haut fonctionnaire de l'Instruction publique et géographe militant, grand champion de l'expansion coloniale. Il a créé une institution hybride, relevant du secteur privé mais bénéficiant dès ses débuts du soutien moral et financier de l'État. Le lien entre les objectifs de l'Alliance et la politique du gouvernement fut indiqué en termes peut-être un peu vagues mais révélateurs par un futur ministre des Colonies, Gaston Doumergue, quand il a déclaré que "l'Alliance française a élargi l'action gouvernementale $»^{7}$. De façon plus explicite, Pierre Foncin reconnaissait dès 1888 qu'une des fonctions importantes de l'Alliance était d'agir à la place du gouvernement dans des circonstances délicates : " elle agit à sa place, là où il risquerait de se compromettre $»^{8}$.

Le premier objectif de l'Alliance fut le rayonnement de la langue française dans les colonies et, accessoirement, à l'étranger. L'école était son principal - voire unique moyen d'action à ses débuts. Elle ciblait l'ensemble de la population scolaire des possessions coloniales, afin de l'intégrer dans une plus grande France. Cette vision était marquée par l'assimilationnisme ambiant de l'époque, la méthode de financement et de gestion relevant du paternalisme.

Le savoir constituant la clé du progrès, Jules Ferry était convaincu que la France avait le devoir de porter la technologie, les sciences et la culture aux pays " arriérés » du globe ${ }^{9}$. A quelques exceptions près, l'appréciation de la culture de l'Autre n'était guère à l'ordre du jour. Bien qu'homme de gauche, Jules Ferry partageait la conviction de l'immense majorité de ses contemporains blancs que la race blanche et sa civilisation étaient supérieures à toutes les autres races et à toutes les autres civilisations, en y ajoutant toutefois l'idée républicaine de l'obligation morale de la mission civilisatrice : «Les races supérieures ont [...] le devoir de civiliser les races inférieures »".

La doctrine de l'assimilation était en harmonie avec ce qu'Albert Salon devait appeler un siècle plus tard l'esprit messianique français. ${ }^{11}$ Dès le Moyen Âge la France, fille aînée de l'Église, se considérait tenue de défendre la foi et de porter le christianisme aux peuples païens. Au XVIII siècle, s'engageant dans un nouvel apostolat, la France se 
fit la championne des Lumières, tandis qu'au lendemain de la Révolution, au nom de la " solidarité des peuples ", elle a cru devoir répandre dans le monde entier les bienfaits de 1789. Ce prosélytisme présupposait une croyance à l'identité fondamentale de l'humanité sous tous les climats et dans tous les temps, ainsi qu'à l'universalité du génie français.

Quoique l'enthousiasme de Ferry pour la version républicaine de l'impérialisme français ne fût pas partagé par toute la gauche (par exemple par Georges Clemenceau) ni par toute la droite - en effet l'opposition monarchiste et le parti militaire y voyaient une façon de détourner les énergies nationales de la Revanche ${ }^{12}$ - il y eut néanmoins un consensus national très large sur la mission civilisatrice de la France dans les colonies et à l'étranger. Bien que le " messianisme » français du XIX siècle ait identifié plusieurs domaines non linguistiques (spirituel, scientifique et social) dans lesquels la France avait des réalisations substantielles à offrir aux nations étrangères, le rayonnement de la langue française a toujours occupé parmi ces zones d'influence potentielles une place privilégiée. Albert Salon a montré que le français est à la fois le symbole et le fondement de l'unité nationale ${ }^{13}$ - en effet celle-ci est assise sur le "droit du sol » plutôt que sur le «droit du sang ». C'est donc avant tout le français qui cimente l'unité de la nation et à ce titre il acquiert un statut supérieur pour devenir un objet de culte, une institution protégée et une affaire d'État.

Offrir au monde le français, et avec le français la culture qu'il véhicule, est perçu à la fois comme un devoir patriotique et une obligation morale. Il est d'ailleurs significatif que les mots " rayonnement " et "diffusion ", d'usage si courant dans le contexte du rôle civilisateur de la France, soient malaisés à traduire en anglais. Les pays anglophones ne se sont guère intéressés à la promotion de leur langue pour elle-même. Parlant plus spécifiquement de l'Amérique du Nord, Louis-Jean Calvet estime que " globalement les États- Unis n'ont semble-t-il jamais eu pour objectif prioritaire de diffuser leur culture [...] et leur langue, leur impérialisme a été principalement politique et économique. Et la langue a suivi $»^{14}$. L'analyse de Thierry de Beaucé va dans le même sens ${ }^{15}$.

En 1884, lors d'une conférence prononcée à Albi, un Jean Jaurès encore obscur approuvait le lien entre la promotion du français et l'expansion coloniale, lien qu'il attribuait à juste titre à la toute jeune Alliance française :

L'Alliance a bien raison de songer avant tout à la diffusion de notre langue : nos colonies ne seront françaises d'intelligence et de cœur que quand elles comprendront un peu le français. [...]

Pour la France [...] la langue est l'instrument nécessaire de la colonisation ${ }^{16 .}$

Pendant les premières années après la fondation de l'Alliance française, Pierre Foncin lui-même professait des idées assimilationnistes passablement radicales. En 1887, dans son rapport à l'Assemblée générale de l'Alliance, il qualifia de "fâcheux état de choses " le fait qu'à Tahiti " tous les indigènes savent lire et écrire, mais ils ne lisent et n'écrivent guère que la langue tahitienne. ${ }^{17} \mathrm{Il}$ rendait " l'ancienne imprévoyance de l'administration française en madère coloniale » responsable de cette situation. Il se distancera ultérieurement d'une conception aussi étroite de la mission civilisatrice de la France, tout comme Jules Ferry d'ailleurs, dont les vues ${ }^{18}$ se nuanceront pour s'ouvrir progressivement au concept de ce qu'on appellera bientôt " l'association » et plus tard encore, dans la deuxième moitié du $\mathrm{XX}^{\mathrm{e}}$ siècle, le « dialogue des cultures » : une forme de partenariat entre colonisateurs et colonisés, partenariat dans lequel les traditions, les institutions et la personnalité des indigènes sont plus largement (bien que toujours 
sélectivement) préservées. Vingt-deux ans après son commentaire sur la place du français à Tahiti, Foncin fera preuve de davantage de réalisme en reformulant les objectifs coloniaux de la France : "Si la population de notre pays ne progresse que lentement, nous possédons aux colonies comme un réservoir d'indigènes que l'École peut rapprocher de nous et jusqu'à un certain point franciser. $»^{19}$

L'apparition de théories sociales pessimistes (dont le dénominateur commun était la mise en question de la perfectibilité de l'homme), telle la théorie de la lutte des espèces chez Darwin, les travaux du psychologue Gustave Le Bon ${ }^{20}$, la réflexion sociologique de Jacques Novikow ${ }^{21}$ ou encore les écrits d'Alfred Fouillée sur la psychologie des nations ${ }^{22}$, confirmait le déclin de l'assimilationnisme. Un de ses critiques les plus en vue fut Léopold de Saussure, frère du linguiste Ferdinand de Saussure. Son livre, la Psychologie de la colonisation française dans ses rapports avec les sociétés indigènes ${ }^{23}$, en gestation depuis de nombreuses années, ne fut livré au public que lorsque l'auteur estimait que celui-ci était prêt à en recevoir le message, tout à la fin du XIX siècle. Il contrastait les tendances assimilationnistes de la France avec les méthodes empiriques plus flexibles de colonisateurs prétendument supérieurs, tels les Romains, les Hollandais et les Britanniques. Selon Saussure, "pénétrée de cette idée consacrée par la Révolution, qu'il existe une formule absolue pour faire le bonheur des peuples, formule indépendante des temps et des lieux, la France s'attribue la mission d'en hâter l'avènement [...] $»^{24}$.

Le terme d'association, dont on peut relever sporadiquement l'occurrence dès le XVIII ${ }^{e}$ siècle $^{25}$, n'entre systématiquement dans le vocabulaire du débat colonial que vers l'époque 1900. C'est après la Première Guerre mondiale qu'avec Albert Sarraut, ministre des Colonies, l'association deviendra la doctrine officielle de la colonisation française. ${ }^{26}$ Toutefois les idées qui sous-tendent ce concept commencent à séduire théoriciens et hommes d'action dès 1890. La nécessité d'une nouvelle orientation s'imposa en raison de l'effritement même des tentatives assimilationnistes de la période précédente : franciser les populations indigènes se révélait être une tâche bien plus difficile que les théoriciens ne l'avaient prévu. Dès 1888, Foncin déplorait qu'en Algérie, trente ans après la conquête, sur 300000 enfants en âge scolaire seuls 10000 aient été scolarisés, et que le bilan pédagogique dans les autres territoires d'outre-mer n'ait pas été meilleur ${ }^{27}$.

Malgré les progrès des thèses associationnistes entre 1890 et 1925, il y a une véritable continuité de la politique linguistique française pendant le dernier quart du XIX ${ }^{\mathrm{e}}$ siècle et au XXe siècle. En 1901, le comte d'Haussonville disait encore qu'il y avait un

...intérêt [...] national à ce que le français continue d'être parlé et compris par le plus grand nombre d'individus possible. Or, proportionnellement du moins, ce nombre diminue, puisque le nombre de ceux qui parlent les langues rivales augmente plus rapidement. Il y a dix-sept ans que l'Alliance française a vu ce péril et s'est constituée pour y porter remède ${ }^{28}$.

Nous retrouvons le même état d'esprit dans une déclaration de Henri Gautier, chef du cabinet du ministre français de l'Instruction publique, au Congrès international de l'enseignement colonial en 1931 : «Faites que chaque enfant né sous notre drapeau, tout en restant l'homme de son continent, de son île, de sa nature, soit un vrai Français de langue, d'esprit et de vocation $! »^{29}$.

Aucune nation n'attache autant d'importance et de valeur à la propagation de sa langue que la France. Aussi la diffusion du français, qu'on pourrait à première vue soupçonner de n'être qu'un outil de domination politique ou commerciale, finit-elle par devenir un objectif en soi. On apprendra donc sans surprise que les dépenses publiques en faveur 
de la promotion de la langue et de la culture françaises dépassent souvent les avantages économiques qui pourraient en découler.

Bien que le fondateur de l'Alliance, qui était aussi le fondateur de la première société de géographie commerciale ait souvent répété que « tout client de la langue française est un client naturel des produits français $»^{30}$, et même que « c'est e $n$ grande partie [...] pour le commerce que travaille l'Alliance française et que nous l'avons fondée $",{ }^{31} \mathrm{en}$ réalité le coût financier de la propagation du français et de la culture française dépassait fréquemment le rendement économique réel ou potentiel de tels investissements. Dire que « la propagation de la langue française est la clef des marchés extérieurs $\|^{32}$ correspondait davantage à une stratégie de sollicitation de subventions auprès du secteur privé qu'à un objectif authentique ou à une projection réaliste. C'est qu'en effet, au-delà de ces déclarations destinées aux mécènes en puissance, les racines profondes de l'Alliance étaient morales et affectives plutôt qu'utilitaires ${ }^{33}$.

Il y a là une véritable contradiction dans la pensée de Pierre Foncin. En effet, il reconnaissait simultanément que « l'Alliance française a été fondée par des hommes d'étude, elle a été surtout propagée jusqu'ici par des professeurs. [...] Elle s'est recrutée principalement parmi ceux qui aiment la langue française pour elle-même, qui ont le loisir de la réflexion et du travail intellectuel pur $»^{34}$.

\section{Pierre Foncin : l'homme et son milieu}

Tout porte à croire que si Jules Ferry n'a pas personnellement participé à l'établissement de l'Alliance française, ses aspirations se reflètent néanmoins dans les principes qui ont présidé à sa création. ${ }^{35}$ C'est Pierre Foncin, le premier secrétaire général de l'Alliance, qui maria les deux aspects fondamentaux de la politique de Ferry, l'expansion coloniale et l'enseignement gratuit et obligatoire, dans l'institution qu'il venait de créer.

Professeur de géographie, voire un des pionniers, avec Paul Vidal de la Blache, de cette discipline en France ${ }^{36}$, Foncin avait été nommé directeur général de l'enseignement secondaire par Paul Bert pendant le bref passage de celui-ci au gouvernement comme ministre de l'Instruction publique ${ }^{37}$. Leur association continua au sein de l'Alliance française jusqu'à la nomination de Bert à un poste de gouverneur colonial. Foncin haut fonctionnaire exerça une influence profonde sur l'enseignement en France à la fin du $\mathrm{XIX}^{\mathrm{e}}$ et au début du XX $\mathrm{XX}^{\mathrm{e}}$ siècle.

Ce petit-fils d'aubergiste et fils d'universitaire (la carrière de Joseph, son père, présente plus d'une analogie avec la sienne ${ }^{38}$ ) était un produit typique du XIX ${ }^{\mathrm{e}}$ siècle français. Né à Limoges en 1841, il suivit son père de ville en ville dans ses différentes affectations de professeur. Normalien (comme Joseph) à 19 ans, agrégé d'histoire à 22 ans, Pierre Foncin enseigna aux lycées de Carcassonne (ville qui lui fournit le sujet d'une de ses thèses, sa thèse majeure portant sur Turgot), de Troyes, de Mont-de-Marsan et de Bordeaux (1869). Nommé chargé de cours de géographie commerciale à la faculté des lettres de Bordeaux, il obtient son doctorat en 1876 et un an plus tard prend possession d'une chaire de géographie créée pour lui à la même faculté. En 1879 il est nommé recteur de l'académie de Douai. ${ }^{39}$ C'est en novembre 1881 qu'il est nommé directeur de l'enseignement secondaire. L'année suivante il devient inspecteur général de l'Instruction publique, fonction qu'il conservera pendant trois décennies, jusqu'à son départ à la retraite en 1911. 
En 1872 il publia un manuel d'histoire pour l'enseignement primaire, Textes et récits de l'histoire de France ${ }^{40}$, qui souleva une énergique opposition dans les milieux conservateurs (notamment de la part de Mgr Dupanloup) - ce manuel ne sera autorisé que cinq ans plus tard ${ }^{41}$ Par la suite le nom de Pierre Foncin sera connu du grand public comme celui de l'auteur de nombreux manuels de géographie dont il se serait vendu, jusqu'en 1920, 26 millions d'exemplaires ${ }^{42}$.

En l'absence d'une biographie de ce champion injustement négligé du rayonnement de la France et de la langue française dans les colonies et à l'étranger, nous en sommes réduits, pour deviner ce que fut l'homme, aux notices des encyclopédies et des dictionnaires biographiques, ainsi qu'à de rares esquisses dans des ouvrages spécialisés ${ }^{43}$ et à quelques bribes de témoignage recueillies au hasard des lectures.

Les allocutions et les communiqués de Foncin le révèlent modéré et raisonnable. Nous avons un rare témoignage confirmant l'image d'une personnalité courtoise et aimable, voire d'une grande humanité, dans les réminiscences de Raoul Blanchard. Celui-ci se souvient d'avoir sollicité l'appui de Foncin, alors inspecteur général, pour sa nomination à un nouveau poste au lycée de Lille. Foncin lui donna rendez-vous à la gare de Saint-Quentin, où il l'attendait « souriant dans sa barbe fleurie ». Il l'emmena visiter le musée Latour, l'invita à déjeuner au meilleur restaurant de la ville et " poussa même la gentillesse jusqu'à [le] reconduire à [son] train ", non sans avoir d'abord confirmé sa nomination à la chaire postulée au Lycée de Lille. Blanchard ajoute : « J'étais éperdu de reconnaissance à l'égard des manières délicates de mon chef ; je me permets de recommander les méthodes Foncin à messieurs les inspecteurs généraux $»^{44}$.

Le triple engagement passionné de Foncin en faveur de l'enseignement, pour l'expansion coloniale et dans le mouvement pour la promotion de la géographie est inséparable de la création de l'Alliance française.

Les sociétés de géographie ${ }^{45}$, dont la première, la Société de géographie de Paris, avait été fondée en 1821, ont connu un vif succès auprès du public français pendant les années soixante-dix et quatre-vingts. Il y a eu à cette époque une véritable prolifération de ces sociétés dont certaines ne cachaient pas leur caractère utilitaire. Ainsi une "Commission de géographie commerciale " avait été établie au sein de la Société de géographie de Paris dès 1873, et une "Société de géographie commerciale ", la première de France, a été formée à Bordeaux en 1874 par Pierre Foncin lui-même ${ }^{46}$. Ces sociétés préconisaient non seulement les voyages et l'exploration désintéressée, mais aussi la colonisation et le développement des territoires conquis. Un des historiens de la société de Bordeaux observe que cette orientation pragmatique des sociétés de géographie constituait « une conjonction assez rare à l'époque entre l'Université et le monde des affaires $»^{47}$, conjonction qui remplirait d'aise bien des présidents d'université d'aujourd'hui... Quoi qu'il en soit, le lien entre la promotion de la géographie et la nouvelle Alliance française à travers la personne de Pierre Foncin est particulièrement significatif.

A la suite de Charles-Robert Ageron $^{48}$, Dominique Lejeune fait remarquer que les sociétés de géographie « s'alignent, dans le cadre d'un parti colonial français, aux côtés d'associations nombreuses et fort variées au sein desquelles on retrouve souvent les mêmes hommes : Alliance française (1883), Société française de colonisation et d'agriculture coloniale, Comité d'Afrique française (1890), etc. ${ }^{49}$. En effet, dès 1978 Ageron avait suggéré que le " parti colonial, c'était surtout pour le public une 
multitude d'organisations et d'associations [...dont] la première et la plus connue [...] puisqu'elle vit toujours, l'Alliance française, fut créée en 1883 par des "coloniaux" convaincus : Paul Bert, V. Duruy, P. Foncin, Ferdinand de Lesseps ${ }^{50}$.

La motivation coloniale de Foncin était d'ailleurs intimement liée à ses préoccupations de démographe : "Puisque la France n'a plus assez d'enfants, il lui reste un moyen d'augmenter la famille nationale : c'est d'adopter et de franciser des races nouvelles ${ }^{51}$. Ou encore :

Puisque [la France] manque d'enfants, qui l'empêche de franciser les fils de ses sujets et protégés coloniaux ? [...] Le premier effet de cette annexion morale doit être l'enseignement de notre langue. [...Celui-ci] est nécessaire dans nos domaines coloniaux [...] parce qu'il est la préface, le commencement indispensable de toute annexion morale. Et cette annexion morale elle-même, nous l'appellerons de tous nos vœux. Pourquoi ? Parce qu'elle achèvera et consolidera nos conquêtes matérielles, parce qu'elle accroîtra artificiellement notre race. Quand un ménage stérile désire des enfants, il en adopte. Eh bien! en attendant que nous ayons retrouvé la fécondité de nos pères [...], adoptons aussi, créons-nous aux quatre coins du globe une grande famille adoptive $[. . .]^{52}$.

Notons que le désir exprimé par Foncin d'« adopter » les indigènes présuppose une certaine affinité avec eux, ou du moins un certain respect pour leur personne, affinité et respect fort éloignés du mépris que la majorité des blancs vouaient à l'époque aux populations colonisées. Le fondateur de l'Alliance française fut dès l'abord moins dédaigneux des races que d'autres ont pu appeler « inférieures » : cette description ne faisait pas partie de son discours. Foncin proposa d'intégrer les indigènes dans la nation française comme membres adoptifs de la famille nationale. Comme tant d'autres apôtres de l'expansion coloniale, il y voyait pour la France une nouvelle source d'énergie et de créativité.

\section{Les débuts de l'Alliance française de Paris}

\section{La fondation de l'Alliance française}

Selon le récit de Pierre Foncin,

il y a un peu plus de vingt-cinq ans ${ }^{53}$, nous étions neuf autour d'une table [...]. Un

diplomate [...] incomparable, M. Paul Cambon, alors résident général en Tunisie, nous avait réunis pour constituer un Comité capable de transmettre une subvention ministérielle à ses chères écoles africaines. C'est dans ce Comité [...] que naquirent l'idée et le nom de l'Alliance française ${ }^{54}$.

La modestie de Foncin lui a interdit d'ajouter que le nom comme le concept étaient de son invention : «le premier, M. Foncin parla d'alliance ${ }^{55}$. Il semble avoir fait sien le projet de Paul Cambon presque immédiatement, non sans en avoir d'abord élargi la portée. Quant au nom "Alliance française ", il a été calqué par Foncin sur celui de $1^{\prime}$ « Alliance israélite universelle », créée à Paris en $1860^{56}$.

Le point de départ paraît donc avoir été géographiquement très limité, mais il est à peu près certain que dès avant la fin de cette réunion du 21 juillet 1883 Pierre Foncin a fait adopter le principe de l'élargissement du projet de la seule Tunisie à l'ensemble des possessions coloniales de la France et que l'idée même d'une Alliance française mondiale a été envisagée ${ }^{57}$. 
Foncin présidera aux destinées de l'Alliance française pendant plus de trente ans, de 1883 jusqu'à la veille de la Première Guerre mondiale, d'abord comme secrétaire général et ensuite comme président, avec une courte période intermédiaire comme vice-président ${ }^{58}$.

Après l'approbation de l'association par le gouvernement ${ }^{59}$, une assemblée générale tenue le 10 mars de la même année dans les locaux de la Société de géographie de Paris $^{60}$ en adopta les statuts. Cette date serait, selon l'historien officiel de l'Alliance, celle de sa "fondation véritable ${ }^{61}$. Le choix du $1^{\mathrm{er}}$ juin $1909^{62}$ pour les fêtes du vingtcinquième anniversaire de l'Alliance confirme 1884 comme la date officielle de sa fondation ${ }^{63}$.

Deux ans et demi plus tard, le 23 octobre 1886, l'Alliance fut reconnue « établissement d'utilité publique ", quatrième étape dans le processus de son établissement, cette étape ultime comportant divers avantages fiscaux et administratifs ainsi qu'un prestige moral accru.

Les objectifs que l'Alliance française s'était fixés lors de sa fondation méritent réflexion, moins comme des indicateurs de la direction qu'elle allait suivre que comme le témoignage des intentions de ses fondateurs, intentions dont elle allait d'ailleurs s'écarter assez rapidement.

Les fondateurs de l'Alliance l'ont définie comme une « Association Nationale pour la propagation de la langue française dans les colonies et à l'étranger ».

Voici la liste des principales activités qu'ils lui ont assignées :

-fonder et subventionner des écoles françaises, ou introduire des cours de français dans les écoles qui en sont dépourvues ;

-former des maîtres, au besoin en créant des Écoles normales ;

-distribuer des récompenses propres à assurer la fréquentation des écoles ;

-donner des prix ou des bourses de voyage aux meilleurs élèves ;

-encourager les publications pouvant seconder l'œuvre de l'Alliance et, en particulier, celles qui ont un caractère pédagogique ;

-publier un Bulletin périodique ;

-organiser des conférences et autres moyens divers de propagande ${ }^{64}$.

Ces indications, interprétées à la lumière de l'évolution ultérieure de l'Alliance, appellent un certain nombre de commentaires.

De toute évidence, dans l'esprit des fondateurs, l'accent devait être mis sur :

-la vocation coloniale de l'Alliance (même si la propagation du français dans les pays étrangers est également mentionnée)

-l'action à travers le système scolaire (ciblant la population en âge scolaire)

-la diffusion de la langue (plutôt que de la langue et de la culture) ${ }^{65}$.

Chacune de ces priorités sera revue et modifiée au cours des années suivantes.

Dans le sous-titre de l'Alliance (Association Nationale pour la propagation de la langue française dans les colonies et à l'étranger), les colonies ont la place d'honneur. Comme nous l'avons déjà vu, la nomination de Pierre Foncin comme secrétaire général confirme cette impression de la priorité que la jeune Alliance accordait à la propagation de la langue française dans les colonies d'Afrique, d'Asie et du Pacifique. Foncin, un des représentants les plus dynamiques de la géographie appliquée, était 
intellectuellement et professionnellement engagé dans la lutte pour l'expansion coloniale.

L'autre qualité de Foncin, celle d'inspecteur général de l'Instruction publique, n'est pas étrangère au rôle primordial que l'école occupait dans la stratégie de l'Alliance française nouvellement fondée. Ce rôle n'est nulle part mieux illustré que dans le programme esquissé par Paul Dupuy dans une conférence prononcée en 1886 sur « Les deux premières années de l'Alliance française »:

Par l'école, vulgariser l'usage du français dans nos colonies et nos protectorats; par l'école, soutenir le prestige de la France dans les contrées barbares [sic] où il domine depuis longtemps, particulièrement dans les pays musulmans du Levant ; par l'école, conserver à la littérature et à la langue françaises leur suprématie dans les pays slaves et latins qui se réclament encore de nous comme de leurs éducateurs; par l'école, garder à la nationalité française ceux de nos concitoyens qui habitent l'étranger, quelque infimes que soient leurs groupes; par l'école, maintenir ou étendre les frontières de la langue française là où elles ne coïncident pas avec nos frontières politiques ${ }^{66}$.

En 1893, dix ans après l'établissement de l'Alliance, alors que la priorité coloniale cédait déjà à une action plus large à l'étranger, le président d'alors, le général Parmentier, expliquait ce que l'objectif scolaire signifiait en termes pratiques :

Quant à nos moyens d'action, l'Alliance se met en rapport avec toutes les écoles françaises à l'étranger, qu'elles soient dirigées par des religieux ou par des laïques, des Français ou des indigènes; elle leur envoie des livres d'étude, du matériel scolaire, des subventions en argent; elle encourage les maîtres et les élèves par des récompenses, des livres, de[s] prix, des médailles; elle s'efforce de créer des cours de français dans les écoles qui en sont encore dépourvues; elle les aide à trouver des professeurs ou leur en envoie, et fonde au besoin elle-même des écoles $[. . .]^{67}$.

Quant à l'efficacité de cette action, Denise Bouche conclut son analyse du rôle de l'Alliance française au Sénégal par la constatation qu'en l'absence de ressources substantielles l'Alliance n'a pu avoir qu'une influence marginale : « Les écoles de postes ou de mission, encouragées par le comité de l'Alliance française [de Saint-Louis] auraient aussi bien existé sans lui $»^{68}$.

La stratégie visant les populations coloniales en âge scolaire n'a pas été non plus sans ses critiques. Ainsi en 1888 La Dépêche de Brest estimait que les ressources de l'Alliance auraient été plus utilement employées à "l'instruction des tirailleurs sénégalais » qu’à l'enseignement du français aux enfants des écoles locales :

Nous pensons que cette mesure produirait des résultats autrement féconds que ceux que l'on compte retirer du fonctionnement de ces écoles de gamins indigènes qui ont été créées en 1884, dans trois ou quatre de nos postes du haut Sénégal, grâce aux subsides de l'Alliance française ${ }^{69}$.

Les priorités de l'Alliance française à ses débuts sont clairement illustrées dans le rapport que Pierre Foncin présenta à l'Assemblée générale du 24 février 1887, soit donc moins de quatre ans après la fondation de l'Alliance. A part les pages consacrées aux activités de l'Alliance en métropole (sous la responsabilité de la "Commission générale de propagande $»^{70}$, les dix pages traitant de ce que le secrétaire général appelait « l'action au dehors » étaient réparties dans les proportions suivantes :

1. Possessions extérieures, colonies et pays de protectorat... plus de cinq pages

2. Pays de civilisation orientale ... un peu plus d'une page

3. Groupes de Français à l'étranger ... un peu moins d'une page

4. Pays de langue française ... moins de deux pages

5. Pays étrangers de civilisation européenne ... une page. 
Au moment de cette Assemblée générale de 1887, les activités de l'Alliance dans les pays de civilisation occidentale (non francophone) n'occupaient donc que $10 \%$ du rapport entier, proportion qui va changer radicalement - et avec une étonnante rapidité pendant les années suivantes. Créateur de l'enseignement obligatoire, gratuit et laïque, Jules Ferry, tout comme Gambetta avant lui, était résolu à ne pas exporter dans les colonies ou à l'étranger les querelles de l'Église et de l'État ${ }^{71}$. Foncin lui-même se trouvait proche de Feny sur le plan philosophique - en 1902, au plus virulent du conflit entre l'Église et l'État, il deviendra le président fondateur de la " Mission laïque française $»^{72}$ - mais en sa qualité d'animateur de l'Alliance française ce franc-maçon, ce champion de l'idéologie républicaine semble avoir soigneusement observé une règle de neutralité absolue ${ }^{73}$. Il a généralement soutenu les écoles congréganistes ${ }^{74}$, tout comme d'ailleurs les établissements scolaires protestants et les écoles de l'Alliance israélite universelle. Pourtant la documentation dont nous disposons actuellement ${ }^{75}$ ne permet pas d'affirmer péremptoirement que la laïcisation imposée par l'État après les élections de 1902 n'a pas eu d'effet sur la politique scolaire de l'Alliance.

\section{Déclin des priorités coloniales et scolaires}

L'historien officiel de l'Alliance, Maurice Bruézière, suggère que la baisse dans les activités de l'Alliance dans les colonies vers la fin du siècle est liée à l'intervention pédagogique plus active de l'État dans ces territoires :

L'Alliance, au début de la colonisation, avait tenté de suppléer à la carence des services officiels de l'Instruction publique dans les territoires nouvellement conquis ; mais à mesure que ces services (en Tunisie, au Maroc, à Madagascar notamment) vont prendre la relève, l'Alliance aura tendance à s'effacer et à ne plus jouer qu'un rôle d'auxiliaire ${ }^{76}$.

En 1909 un service spécial des "Écoles françaises dans le monde " a été créé, qui deviendra l'année suivante l'office national des universités et écoles françaises ${ }^{77}$. Cette initiative soulagera l'Alliance d'une de ses plus grosses responsabilités, ou, si l'on préfère, elle lui ôtera une de ses raisons d'être.

Il se fait que le déclin de l'importance de l'école dans l'action de l'Alliance a grosso modo coïncidé avec la popularité croissante des cours d'été ${ }^{78}$ en métropole et des classes de français pour adultes dans les pays dits de civilisation occidentale. Si la chronologie exacte de cette mutation reste encore à établir, les données que nous avons relevées dans le Bulletin de l'Alliance française, le livre de Maurice Bruézière et la thèse d'Albert Salon permettent de suggérer que ce processus avait commencé très tôt après la fondation de l'Alliance et qu'il a été mené à terme dans les toutes premières années du XXe siècle.

Dès 1893 l'Assemblée générale a exprimé le désir

que notre Association, après avoir appelé l'attention du gouvernement sur la nécessité de propager le français dans les colonies, lui cède bientôt entièrement la place en ce domaine, afin de reporter toute l'énergie de son action dans les pays étrangers proprement dits ${ }^{79}$.

L'expression qui suit, toutefois, à savoir « qui sont en vérité son naturel et seul département » représente un écart radical par rapport aux premiers objectifs de l'Alliance.

En effet, six ans plus tôt Pierre Foncin avait conclu son rapport à l'Assemblée générale du 24 février 1887 par la déclaration suivante : 
Je terminerai la revue de nos travaux par l'indication des faits accomplis dans les pays de civilisation européenne parlant une langue différente de la nôtre. Là, il semble au premier abord que l'Alliance française n'ait point à intervenir.

La confrontation de ces deux textes, l'un datant de 1887, l'autre de 1893, met bien en évidence le changement substantiel intervenu dans les objectifs de l'Alliance pendant les dix premières années de l'existence de l'association, les priorités coloniales du début cédant la place à l'action dans le monde dit « occidental».

Ce n'était pas la seule mutation. A ses débuts, l'Alliance avait mis l'accent presque exclusivement sur l'enseignement de la langue. Progressivement, à cette priorité s'est substituée une conception plus éclectique des objectifs de l'Alliance, englobant désormais également la culture, dans toute la richesse de ses manifestations (littérature, théâtre, musique, beaux-arts, etc. ${ }^{80}$. Cette dernière mutation n'était pas étrangère à l'affaiblissement des priorités coloniales et scolaires dans l'action de l'Alliance.

Les fondateurs avaient investi beaucoup d'énergie dans l'établissement de branches en métropole, plus particulièrement à Paris. Elles avaient pour tâche de collecter des fonds pour subventionner les activités de l'Alliance à l'étranger et surtout dans les colonies : « l'une des fonctions essentielles de notre Association [...] est de recueillir en France le plus d'argent possible afin d'en avoir le plus possible aussi à distribuer hors de France $»^{81}$. Un autre objectif important des branches métropolitaines était de faciliter l'accueil des étudiants étrangers en France. Pour maintenir l'enthousiasme des membres en métropole, l'Alliance organisait régulièrement à leur intention, à titre de " propagande ", des conférences, des causeries, des débats et autres activités culturelles. Autour de 1900 un nombre croissant de branches métropolitaines se trouvaient en déclin. Il en était de même des branches coloniales - selon Bruézière, en raison du manque de stabilité de l'encadrement par le colonisateur ${ }^{82}$, l'Alliance à ses débuts était incontestablement une institution paternaliste. Il ressort également du livre de Maurice Bruézière ${ }^{83}$ que ces fondements paternalistes - comme par exemple la tradition selon laquelle les branches métropolitaines avaient pour mission de subventionner les branches coloniales et étrangères - s'affaiblissaient avec le refroidissement des enthousiasmes en métropole et les mutations dans le champ d'activité de l'Alliance.

L'Alliance, telle qu'en elle-même son évolution l'a changée, était désormais mieux adaptée aux besoins de régions où les adhérents étaient économiquement indépendants et spontanément motivés, c'est-à-dire les pays européens et l'Amérique du Nord. Les droits et responsabilités des comités régionaux qui bénéficiaient dans une large mesure de l'autonomie financière et administrative ${ }^{84} n$ 'ont pas été définis du jour au lendemain : ainsi par exemple à l'Assemblée générale de 1887 Pierre Foncin évoquait le cas de Prague où une association déjà existante s'était rebaptisée "Alliance française de Prague » et reçut des livres du comité de Paris. Selon le président de Prague, « bien que nul rapport de subordination quelconque ne nous lie à elle, bien que notre autonomie soit aussi entière qu'elle peut l'être, l'Alliance de Paris nous regarde comme sienne [...] $»^{85}$.

Les statistiques publiées par Albert Salon illustrent le déséquilibre en ressources qui existait entre les Alliances des diverses régions du monde - et nous pouvons présumer que dans l'ensemble les ressources reflètent le niveau d'activitée ${ }^{86}$.

Ressources des alliances par région (1883-1918) 


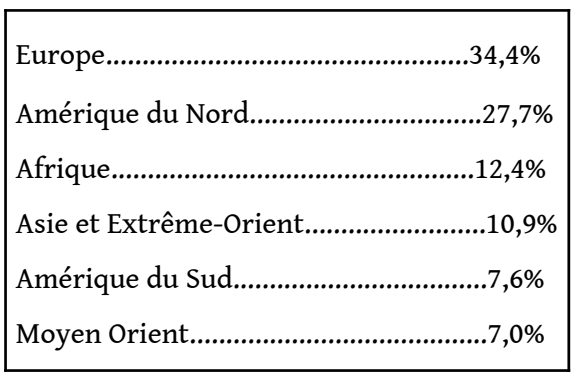

Ces chiffres comprennent les subventions de Paris (un tiers du total) et les ressources locales (deux tiers du total), mais bien entendu la part des subventions varie d'une région à l'autre :

Subventions de Paris (comme proportion des ressources totales disponibles dans une région donnée)

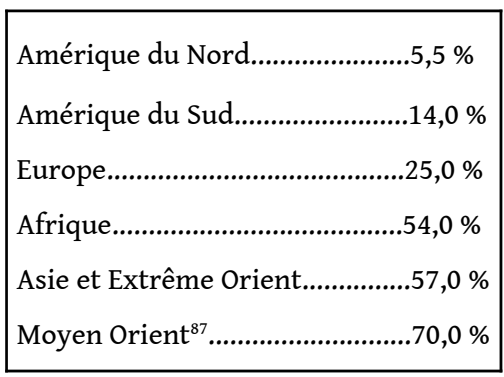

A la veille de la Première Guerre mondiale, le secteur le plus dynamique de l'Alliance était celui des classes pour adultes ${ }^{88}$, avec les diverses activités culturelles et sociales qui y étaient associées, tandis que l'objectif original de 1883-84 de dispenser un enseignement de masse dans les écoles coloniales avait beaucoup perdu de son élan ${ }^{89}$. Les branches connaissant la croissance la plus rapide étaient celles d'Europe et d'Amérique du Nord. Une circulaire signée du président (Pierre Foncin), du viceprésident et du secrétaire général ${ }^{90}$ désigna les comités de Copenhague, de Stockholm, de Prague, de Moscou et ceux des États-Unis comme « les plus actifs, les plus brillants ", tous gérés essentiellement par les citoyens des pays d'accueil. Tel était, après vingtdeux ans, le véritable visage de l'Alliance française.

\section{Conclusion}

En attendant la rétrocession à la France des archives de l'Alliance française par la Russie, nous avons tenté, avec la documentation actuellement disponible, d'esquisser le contexte idéologique et politique dans lequel l'Alliance a vu le jour dans le dernier quart du XIX ${ }^{e}$ siècle et de tracer les mutations qu'elle a connues pendant les trente premières années de son histoire. Toute cette période - l'établissement de l'Alliance et les changements qu'elle a su opérer - est placée sous le signe de son fondateur, Pierre Foncin.

Avec une entorse à la clarté cartésienne et contrairement aux traditions jacobines de la République, l'Alliance française a fini par se doter d'un modèle d'organisation décentralisé, pluraliste mais quelque peu ambigu qui s'est révélé suffisamment flexible pour s'adapter aux besoins des différentes régions du monde. Si ce modèle, 
particulièrement approprié aux pays de type occidental, ne se situe pas dans l'axe principal de la tradition républicaine, il est par contre en harmonie avec les tendances décentralisatrices de Pierre Foncin. En effet, le secrétaire général, fort de son expérience personnelle ancrée dans les provinces françaises, ainsi que de sa formation d'historien et de géographe, a toujours soutenu la cause du régionalisme ${ }^{91}$.

Les facteurs que nous avons passés en revue, à savoir l'évolution des attitudes en matière coloniale, les difficultés auxquelles les premiers efforts scolaires de l'Alliance se sont heurtés dans les colonies, le rôle plus actif que l'État était amené à jouer dans l'enseignement colonial, la réussite croissante des branches de l'Alliance dans les pays de civilisation occidentale, la meilleure adéquation de celles-ci aux structures financières et administratives de l'institution, l'intérêt que les adhérents de ces pays portaient aux activités culturelles (au delà et en plus de l'enseignement de la langue), le succès des cours d'été pour étudiants étrangers en métropole, tous ces facteurs ont poussé l'Alliance française de Pierre Foncin à repenser ses objectifs et ses priorités.

Ces mutations - qui commençaient à se manifester à partir de 1890 - seront expressément assumées à partir de 1893, dix ans après la fondation de l'Alliance. Certes, l'institution ne se détournera jamais complètement de l'action dans les colonies et les ex-colonies, ni des établissements scolaires, mais les priorités coloniales et scolaires n'en continueront pas moins à décliner jusqu'à la Première Guerre mondiale, 1914 étant d'ailleurs la date du départ de Foncin de la direction de l'Alliance. Il mourra deux ans plus tard.

Ces mutations, loin d'être une trahison de la première vocation de l'Alliance française, en sont une réinterprétation à la lumière de besoins nouveaux. Elles témoignent de la lucidité de son fondateur, jusque dans l'automne de sa vie, ainsi que du dynamisme et de la vitalité de l'institution.

BIBLIOGRAPHIE

\section{Périodiques}

$B A F$ : Bulletin de l'Alliance française (Paris).

\section{Livres, thèses, mémoires, chapitres de livres, articles, etc.}

AGERON, Charles Robert (1978), France coloniale ou parti colonial ? Paris, Presses Universitaires de France.

BEAUCE, Thierry de (1988), Nouveau discours sur l'universalité de la langue française, Paris, Gallimard.

BETTS, Raymond F. (1961), Assimilation and Association in French Colonial Theory, 1890-1914, New York, Columbia University Press. 
BOUCHE, Denise (1975), « L'Enseignement dans les territoires français de l'Afrique occidentale de 1817 à 1920 - Mission civilisatrice ou formation d'une élite ? ", thèse présentée devant l'Université de Paris I le 8 juin 1974, 2 tomes, Service de reproduction des thèses, Université de Lille III.

BOUCHE, Denise (1991), Histoire de la colonisation française, Tome second, Flux et reflux (1815-1962), Paris, Fayard.

BROC, Numa (1976), « Patriotisme, régionalisme et géographie : Pierre Foncin (1841-1916) », L'Information historique, $38^{\mathrm{e}}$ année, pp. 30-33.

BRUEZIERE, Maurice (1983), L'Alliance française - Histoire d'une institution, Paris, Hachette.

CONKLIN, Alice L. (1997), A Mission to Civilize - The Republican Idea of Empire in France and West Africa, 1895-1930, Stanford, Stanford University Press.

GAILLARD, Jean-Michel (1989), Jules Ferry, Paris, Fayard.

GANIAGE, Jean (1968), L’Expansion coloniale de la France sous la Troisième République (1871-1914), Paris, Payot.

GIRARDET, Raoul (1972), L'Idée coloniale en France de 1871 à 1962, Paris, La Table ronde.

LEJEUNE, Dominique (1993), Les Sociétés de géographie en France et l'expansion coloniale au XIXe siècle, Paris, Albin Michel.

LEJEUNE, Dominique (1999), « Les Sociétés de géographie en France, dans le mouvement social et intellectuel du XIX ${ }^{e}$ siècle ", thèse pour le doctorat d'État ès lettres et sciences humaines (histoire), soutenue le 12 juin 1987 à l'Université de Paris X-Nanterre, texte de la version intégrale, arrêté le 7 septembre 1999.

MURPHY, Agnes (1948), The Ideology of French Imperialism - 1871-1881, Washington D.C., The Catholic University of America Press.

SALON, Albert (1981), "L'Action culturelle de la France dans le monde : analyse critique », thèse dactylographiée de doctorat ès lettres, Université de Paris 1 (Panthéon-Sorbonne).

SALON, Albert (1983), L'Action culturelle de la France dans le monde, Paris, Fernand Nathan.

SAUSSURE, Leopold de (1899), Psychologie de la colonisation française dans ses rapports avec les sociétés indigènes, Paris, Alcan.

SPAËTH, Valérie (1999), « La didactique du français aux étrangers dans la seconde moitié du XIX siècle : une comparaison des méthodes destinées aux Européens, aux patoisants et aux colonisés ", Documents pour l'histoire du français langue étrangère out seconde, n² 23, juin 1999, pp. 124-140.

\section{NOTES}

1. Nous tenons à exprimer notre gratitude à Madame Denise Bouche ainsi qu'à MM. Paulin Djité et Antony Walsh, de nous avoir aidé de leurs conseils et critiques dans la préparation de cette étude. Nous sommes également reconnaissant à M. Francis Lecompte, Directeur de l'action à l'étranger à l'Alliance française de Paris, de s'être intéressé à nos recherches et de nous avoir donné accès à un certain nombre d'extraits du Bulletin de l'Alliance française. Il va sans dire que ni Mme Bouche ni MM. Lecompte, Djité et Walsh ne sont en rien responsables des imperfections de ce travail ni des opinions qui y sont exprimées.

2. Désormais BAF. 
3. Bruézière, Maurice (1983), L'Alliance française - Histoire d'une institution, Paris, Hachette. L'auteur est un ancien Directeur de l'École Internationale de l'Alliance. Voir aussi Spaëth, Valérie, « La didactique du français aux étrangers dans la seconde moitié du XIX ${ }^{\mathrm{e}}$ siècle : une comparaison des méthodes destinées aux Européens, aux patoisants et aux colonisés », Documents pour l'histoire du français langue étrangère et seconde (1999 : 23, 124-40).

4. Nous devons cette information à M. Francis Lecompte (communication personnelle du 16 novembre 1998.) Selon une information publiée dans Le Monde du 8 juillet, la totalité des archives françaises transférées de Berlin à Moscou sera prochainement restituée à la France.

5. Maurice Bruézière choisit le 20 mars 188Z, date de l'Assemblée générale constituante de l'Alliance française, comme la véritable date de fondation de l'Alliance, plutôt que la da te de la création du Comité d'organisation le 21 juillet 1883. Voir Bruézière (1983: 10).

6. Parmi ceux-ci une place spéciale revient à la monumentale thèse de Salon, Albert (1981), « L'Action culturelle de la France dans le monde : analyse critique ", thèse dactylographiée de doctorat ès lettres, Université de Paris 1 (Panthéon-Sorbonne), 2040 p. Un des nombreux mérites de cet ouvrage est de contenir des références précises aux archives du ministère des Affaires étrangères.

7. BAF (1903: 141).

8. $B A F(1888: 28)$

9. Voir Biondi, Jean-Pierre (1992), Les Anticolonialistes (1881-1962), Paris, Robert Laffont : 28, ainsi que Baumgart, Winfried (1982), Imperialism - The Idea and Reality of British and French Colonial Expansion, 1881-1914, Oxford, Oxford University Press : 72.

10. Discours du 28 juillet 1885 prononcé à la Chambre des Députés, cité par Brunschwig, Henri (1960), Mythes et réalités de l'impérialisme colonial français - 1871-1914, Armand Colin : 75.

11. Salon, Albert (1983), L'Action culturelle de la France dans le monde, Paris, Fernand Nathan : 31-39.

12. Encourager les Français à reporter leurs énergies sur le domaine colonial cadrait parfaitement avec la politique de Bismark - voir Baumgart (1982: 59).

13. Salon $(1983: 68)$.

14. Calvet, Louis-Jean (1987), La Guerre des langues et les politiques linguistiques, Paris, Payot : 269-70. Notons d'ailleurs que l'expression « la guerre des langues » n'est pas nouvelle : elle a été utilisée dès 1885 , notamment par l'économiste Charles Gide, qui a intitulé une de ses conférences « Lutte des langues à la surface du globe : rôle de l'Alliance française ». Conférence prononcée à Nîmes le 6 juin 1885. Bruézière (1983 : 20). Voir aussi Flaitz, Jeffra (1988), The Ideology of English - French Perceptions of English as a World Language, Berlin, Mouton de Gruyter, Pennycook, Alastair (1994), The Cultural Politics of English as an International Language, London, Longman, et Phillipson, Robert (1992), Linguistic Imperialism, Oxford, Oxford University Press.

15. Beaucé, Thierry de (1988), Nouveau discours sur l'universalité de la langue française, Paris, Gallimard : 116 .

16. Texte cité dans Agron, Charles Robert (1978), France coloniale ou parti colonial ? Paris, Presses Universitaires de France : 156, d'après Jaurès, Jean (1959), Textes choisis, Introduction et notes par Madeleine Revérioux, Paris, Editions sociales.

17. BAF (1887 : 13). Ce que Louis-Jean Calvet appelle la dans son livre (1974) Linguistique et colonialisme - Petit traité de glottophagie, Paris, Payot, c'est bien ces tentatives de suppression des langues autochtones ainsi que la guerre que la République menait (et que certains mènent encore) contre les langues régionales.

18. Voir Gaillard, Jean-Michel (1989), Jules Ferry, Paris, Fayard : 598-612.

19. Souligné par nous. Discours prononcé le 1er juin 1909 en Sorbonne, à l'occasion du 25e anniversaire de la fondation de l'Alliance française, cité dans BAF (1909 : 224).

20. Voir Le Bon, Gustave (1881), L'Homme et les sociétés, leurs origines et leur histoire, 2 vols, Paris, J. Rothschild, et celui de ses livres qui a exercé le plus d'influence, Les Lois psychologiques de l'évolution des peuples, Paris, Félex Alcan, 1ère édition (1894). 
21. En particulier Novikow, Jacques (1893), Les Luttes entre sociétés humaines et leurs phases successives, Paris, Félix Alcan, cité par Betts, Raymond F. (1961), Assimilation and Association in French Colonial Theory, 1890-1914, New York, Columbia University Press : 217.

22. Par exemple Fouillée, Alfred (1894), dans la Revue des deux mondes, (1894 : CXXIV, 76-107). Voir Betts (1961 : 61-64 et 212).

23. Saussure, Léopold de (1899), Psychologie de la colonisation française dans ses rapports avec les sociétés indigènes, Paris, Alcan. Léopold de Saussure reconnaissait volontiers sa dette envers les travaux de Gustave Le Bon.

24. Saussure (1899: 26).

25. Ainsi, Robinet dans son Dictionnaire universel des sciences morales, préconisait dès 1789 l'association entre le colonisateur et l'indigène. Voir aussi le titre du livre du Comte de Laborde (1821), L'Esprit d'association dans tous les intérêts de la communauté sur la situation en SaintDomingue. La première apparition du terme au sens technique moderne sera relevée en 1887 dans une conférence de Jules Harmand sur « L'Indo-Chine française, politique et administration ", prononcée devant l'Association républicaine du Centenaire de 1789. Voir Betts (1961: 194).

26. Voir Conklin, Alice L. (1997), A Mission to Civilize - The Republican Idea of Empire in France and West Africa, 1895-1930, Stanford, Stanford University Press : 187 et sqq.

27. BAF (1988 : 167). Voir aussi Beaucé (1988 : 172-83).

28. BAF (1901: 82).

29. Gautier, Henri (1931), L'Adaptation de l'enseignement aux colonies, [...]. Paris, H. Didier : 293, cité par Bouche, Denise (1991), Histoire de la colonisation française, Tome second, Flux et reflux (1815-1962), Paris, Fayard : 271.

30. Voir, entre autres, $B A F(1888$ :170). En 1901, le secrétaire général de l'époque, L. Dufourmantelle, rappelle à l'Assemblée générale annuelle et préconise. Voir BAF (1901:137).

31. BAF (1888 : 170). Voir aussi la page de Dominique Lejeune où cet historien rappelle l'amalgame que certains avaient tendance à faire entre l'Alliance et l'expansion économique. Ainsi Louis Arnavon, fabricant de savon à Marseille et président-fondateur du comité marseillais de l'Alliance française, selon Lejeune, aurait offert de "mettre au service des négociants les nombreux comités de l'Alliance à l'étranger ». Voir Bulletin de la Société de géographie de Marseille, cité par Lejeune, Dominique (1993), Les Sociétés de géographie en France et l'expansion coloniale au XIXe siècle, Paris, Albin Michel : 151.

32. Pierre Foncin, parlant devant un auditoire d'hommes d'affaires, cité dans BAF (1888:173).

33. Ainsi, selon René Girardet, « dans la pensée de Ferry et des hommes de son école [...] ce n'est pas l'argumentation patriotique qui doit être considérée comme l'alibi des impératifs économiques, mais bien davantage la théorisation économique qui sert à légitimer les desseins nationalistes ». Girardet, René (1972), L'Idée coloniale en France de 1871 à 1962, Paris, La Table ronde : 51 .

34. BAF (1888 : 172).

35. Sur le rôle de Jules Ferry dans l'expansion coloniale voir Bouche (1991 : en particulier 53-57, 207-21 et 243-73).

36. Voir Broc, Numa (1976), « Patriotisme, régionalisme et géographie », L'Information historique : 38, 30-33.

37. Voir Dictionnaire de biographie française, Paris, Letouzey et Ané (1979 : XIV, 258).

38. Selon Broc, « chrétien libéral, passionné de pédagogie, Joseph Foncin a eu sur la vocation de son fils une influence déterminante ». Broc $(1976: 30)$.

39. Dans ces fonctions, il se distingue par ses efforts pour développer l'enseignement secondaire pour les jeunes filles. Voir Broc $(1976: 31)$.

40. Broc (1976 : 331).

41. Lejeune, Dominique (1999 [1987]), « Les Sociétés de géographie en France, dans le mouvement social et intellectuel du XIXe siècle ", thèse pour le doctorat d'État ès lettres et sciences humaines 
(histoire), soutenue le 12 juin 1987 à l'Université de Paris X - Nanterre, texte de la version intégrale arrêté le 7 septembre 1999, chapitre 5.

42. Voir Mollier, Jean-Yves et Jocelyne George (1994), La plus longue des républiques -1870-1940, Paris, Fayard : 313-14.

43. Notamment Dictionnaire de biographie française, Paris, Letouzey et Ané (1979 : XIV, 258) et Lejeune (1999 [1987]).

44. Blanchard, Raoul (1963), Je découvre l'Université, Paris, Arthème Fayard : 54-55. Nous sommes redevable de la découverte de ce témoignage à notre collègue Colins Thomton-Smith.

45. Sur le rôle des sociétés de géographie la thèse de Murphy, Agnes (1948), The Ideology of French Imperialism - 1871-1881, Washington D.C., The Catholic University of America Press : 1-40 a été pendant longtemps l'ouvrage de base. Le livre récent de Lejeune (1993) et surtout la thèse de doctorat d'État du même auteur, thèse sur laquelle le livre est basé, font désormais autorité. Voir aussi, pour une présentation plus sommaire, Girardet (1972:32-35).

46. Voir Lejeune (1993) et Lejeune (1999 [1987] : chapitre 6 et Annexe AA).

47. Broc, Numa (1978), " Le Rôle de la Société de géographie de Bordeaux (1874) dans les premiers congrès nationaux de géographie, 1878-1896, Revue de géographie des Pyrénées et du SudOuest : 49, 150-55, cité par Lejeune (1999 [1987] : Annexe AA).

48. Ageron (1978: 156).

49. Lejeune (1993 : 162). Par «les mêmes hommes » l'auteur entend sans doute, dans le cas de l'Alliance française, Paul Cambon, Paul Bert, Pierre Foncin, Louis Machuel ou encore, parmi les présidents d'honneur, Faidherbe et Ferdinand de Lesseps. Voir Bruézière (1983: 10).

50. Ageron (1978: 156).

51. En italiques dans le texte. Pierre Foncin, allocution prononcée lors d'un spectacle organisé au profit de l'Alliance française au Théâtre du Vaudeville le 2 février 1888, cité dans BAF (1888:26).

52. Conférence citée dans BAF (1888: 166-73).

53. Le 21 juillet 1883, au Cercle Saint-Simon, 215 boulevard Saint-Germain. Voir Bruézière (1983 : 10).

54. BAF (1909: 224). Selon un autre témoignage, Paul Cambon a été immédiatement nommé président du comité tunisien, avec Louis Machuel, le nouveau directeur de l'Instruction publique en Tunisie, comme son vice-président. Voir la conférence déjà citée de Paul Dupuy dans BAF (1886: 82-83).

55. Frank Puaux, vice-président de l'Alliance française de Paris, dans un discours prononcé le 8 novembre 1909 à l'occasion du dîner mensuel de la Société des Gens de Lettres. Voir BAF (1909 : 10).

56. Salon (1983: 85).

57. Sauf indication contraire, toutes les informations sur l'établissement de l'Alliance française sont tirées de Bruézière (1983). Les personnes présentes à la réunion inaugurale de l'Alliance française, à part Pierre Foncin, le futur secrétaire général de l'organisation, étaient le diplomate Paul Cambon, alors résident général de France en Tunisie, Louis Machuel, directeur de l'Éducation en Tunisie, M. Jusserand, haut fonctionnaire au ministère des Affaires étrangères, Alfred Mayrargue, représentant la communauté juive, Paul Melon, représentant la communauté protestante, le Père Charmetant, missionnaire apostolique, et Paul Bert, médecin de formation et chercheur, ancien ministre de l'Instruction publique et futur gouverneur colonial. Par la suite le Comité cooptera d'autres personnalités en qualité soit de "présidents d'honneur » soit de « membres d'honneur ». Dans la première catégorie figureront Hippolyte Carnot, membre du Sénat, le cardinal Lavigerie, archevêque d'Alger, et Ferdinand de Lesseps, le créateur du Canal de Suez ; dans la seconde, des érudits, des savants ou des écrivains tels Octave Gréard, Gaston Maspero, Gaston Paris, Désiré Nisard, Ernest Renan, Hippolyte Taine, le marquis de Vogué et Louis Pasteur. 
58. Secrétaire général de 1884 à 1897 et président de 1899 à 1914, avec une brève période intermédiaire comme vice-président, en attendant la fin du mandat du général Parmentier.

59. Arrêté du ministre de l'Intérieur, 24.1.1884.

60. Lejeune (1993: 164).

61. Bruézière (1983 : 11). Une interprétation différente fut proposée par Pierre Foncin lui-même en 1888 : «L'Alliance française a été fondée en juillet 1883 ; elle a commencé à fonctionner en janvier 1984 [...]. » Voir BAF (1888: 172).

62. Séance solenelle en Sorbonne. Voir BAF (1909: 222).

63. Le conseil d'administration de l'Alliance comprendra 50 membres. Ceux-ci seront choisis avec le plus grand éclectisme pour assurer un maximum d'équilibre idéologique, religieux et professionnel dans sa composition. La Commission exécutive (plus tard "Bureau central ») comprendra le président, quatre vice-présidents, le secrétaire général, le trésorier, le trésorier adjoint, six secrétaires, l'archiviste et le comptable. Charles Tissot, diplomate et membre de l'Institut, fut le premier président de l'Alliance française, avec Paul Bert, Paul Cambon, Victor Duruy historien et ancien ministre), ainsi que le Sénateur Pardieu comme vice-présidents. Pierre Foncin gardera jalousement les prérogatives du Conseil d'administration : « Le dernier mot sur toutes les affaires doit rester et appartient à votre conseil d'administration [...] que vous allez pour la première fois compléter ou partiellement renouveler en procédant à une élection dans les formes prévues par vos statuts [...]». Voir BAF (1885:9).

64. Reproduit d'après Bruézière (1983: 11).

65. L'analyse d'Albert Salon diffère de la nôtre - voir Salon $(1983: 20)$ : il voit la fondation de l'Alliance comme une initiative des géographes pour contrecarrer l'influence de l'Empire allemand dans les pays auxquels ni la République ni les congrégations n'avaient accès, donc les pays autres que les colonies françaises. Son interprétation est antérieure à la publication de l'ouvrage de Bruézière sur l'histoire de l'Alliance (1983).

66. $B A F(1886: 86)$.

67. Discours prononcé le 22 mai 1893 au Congrès de l'Alliance française en Sorbonne. Voir BAF (1893:107).

68. Bouche, Denise (1974), «L'Enseignement dans les territoires français de l'Afrique occidentale de 1817 à 1920 - Mission civilisatrice ou formation d'une élite?», thèse présentée devant l'Université de Paris I le 8 juin 1874, 2 tomes, Service de réproduction des thèses, Université de Lille III : I, 367.

69. $B A F,(1888: 97)$.

70. Présidée depuis le 11 février 1885 par l'éditeur Armand Colin et ciblant essentiellement la collecte de fonds.

71. En fait les futurs objectifs de l'Alliance sont éclairés d'une lumière nouvelle par une note manuscrite de la main même de Jules Ferry, note écrite vers 1881-82, donc deux ans avant la fondation de l'Alliance : « Il ne serait ni utile ni habile, pour le moment, de tenter la fondation en Tunisie soit [...] d'un lycée [...], faisant concurrence au Collège de Mgr Lavigerie, soit d'écoles primaires supérieures laïques, les écoles confessionnelles catholiques et israélites étant en possession de la faveur publique [...]. L'œuvre vraiment politique et civilisatrice à poursuivre serait l'École française pour les musulmans, l'École où les instituteurs arabes professeraient le français pour les Arabes [... afin de] franciser la classe moyenne [...].» Cité par Salon (1981: 151).

72. Salon (1983 : 20). En fait la création de la Mission a été décidée dans les locaux mêmes de l'Alliance française.

73. Le cardinal Lavigerie, archevêque d'Alger, était parmi les partisans les plus chaleureux de l'Alliance française, la défense et l'illustration du français étant une des rares causes sur lesquelles un consensus national fût possible.

74. Il convient à cet égard de distinguer entre les écoles publiques sous le contrôle de l'État mais tenues par des congréganistes et les écoles de mission proprement dites. Celles-ci favorisaient 
souvent l'utilisation des langues indigènes. Sur ces questions voir Bouche (1975). Sur la distribution équitable des subventions voir le rapport du Comte d'Aussonville, cité dans BAF (1901: 82).

75. Nous découvrirons peut-être la réponse à cette question après la rétrocession des archives de l'Alliance française par la Russie. Sur la laïcisation de l'enseignement après 1902, voir Bouche (1975, en particulier 476-96) et Bouche (1991: 249-56 et 271-72).

76. Bruézière (1983: 62).

77. Salon (1983:24).

78. Lancés par Ferdinand Brunot en 1894.

79. BAF (1893:11). Souligné par nous.

80. Voir le sous-titre de l'Alliance ainsi que la liste de ses premiers objectifs.

81. Pierre Foncin à l'Assemblée générale de 1889 , cité dans BAF (1889:116).

82. Bruézière (1983: 63) : « les fonctionnaires et les officiers sont trop souvent mutés pour pouvoir assurer durablement le fonctionnement des groupes dont ils s'occupent ».

83. Bruézière (1983 : en particulier 16-79). L'auteur fournit rarement des renseignements statistiques complets - les chiffres qu'il nous donne couvrent tel ou tel secteur pour telle ou telle période, de sorte qu'il n'est pas aisé d'en tira: des conclusions globales.

84. Leur seule obligation consistait à tenir la maison mère informée de l'utilisation qu'ils faisaient de leurs fonds et autres ressources, quelle que fût l'origine de ces ressources, locale ou parisienne.

85. Voir BAF (1887 : 19).

86. Pourcentage basé sur Salon (1981 : 119-121). Albert Salon se pose la question de savoir si la disproportion serait moindre si la période envisagée s'arrêtait en 1913, plutôt qu'en 1918 - en d'autres termes s'il n'y avait pas eu, pendant les années de guerre, un ralentissement anormal de l'activité dans les pays et territoires d'outre-mer.

87. A l'exclusion de l'Egypte, pays inclus dans le chiffre pour l'Afrique et qui en constitue d'ailleurs une proportion substantielle.

88. Le premier cours d'été, lancé le 9 juillet 1894, fut sanctionné par un « Diplôme d'aptitude à l'enseignement du français » ou, à un niveau plus modeste, par un «Certificat d'assiduité ». Voir Bruézière (1983 : 34-35) et BAF (1896:12-17). Le concept de « cours permanents » a pris plusieurs années à prendre forme. Une des raisons de ce long retard a été la nécessité de disposer de locaux adéquats. La création de l'École pratique de l'Alliance n'a eu lieu qu'après la guerre, en 1919. Voir Bruézière (1983 : 52-58 et 91-93).

89. En 1899 Pierre Foncin a distingué clairement entre deux objectifs différents : " Dans un très grand nombre de contrées [l'Alliance] subventionne ou elle entretient des écoles de français et croit servir ainsi directement les intérêts nationaux ; mais dans la plupart des États de la civilisation occidentale elle se borne à un rôle purement littéraire. [...] Elle contribue à la fondation de cercles de conversation française, de bibliothèques françaises, de sociétés françaises où des étrangers se réunissent pour entendre des lectures et des conférences françaises, pour jouer des pièces françaises, ou même pour danser et s'amuser honnêtement en français ». Voir $B A F(1897: 16)$.

90. $B A F(1906: 5-6)$.

91. Membre de la Fédération régionaliste de France, il fut responsable d'un projet de « fédéralisme administratif » - Foncin, Pierre (1898), « Les pays de France » - qui privilégiera les arrondissements et les régions, aux dépens des départements. Voir Broc (1976 : 30-32). Auteur d'un Guide de la Cité de Carcassome (1900), Pierre Foncin a également publié une monographie régionale sur Les Maures et l'Estérel (1910). 
INDEX

Mots-clés : Alliance française, Pierre Foncin, colonialisme, politique linguistique

Keywords : Pierre Foncin, colonialism, linguistic policy

\section{AUTEUR}

IVAN BARKO

Université de Sydney 